\title{
IMPLANTAÇÃO DE SISTEMA DE NOTIFICAÇÃO ELETRÔNICA DE TECNOLOGIAS EM SAÚDE ${ }^{1}$
}

\section{IMPLEMENTATION OF ELECTRONIC NOTIFICATION SYSTEM TECHNOLOGIES IN HEALTH}

\section{APLICACIÓN DE TECNOLOGÍAS ELECTRÓNICAS DEL SISTEMA DE NOTIFICACIÓN EN SALUD}

\author{
NATASHA DEJIGOV MONTEIRO DA SILVA \\ Universidade Uninove \\ natasha.dejigov@hc.fm.usp.br
}

\author{
ANTONIO PIRES BARBOSA \\ Universidade Uninove
}

rbe.pires@gmail.com

\section{RESUMO}

O Complexo HCFMUSP, por ser parte da Rede Sentinela da ANVISA desde 2001, assumiu o compromisso de colaborar na vigilância pós-comercialização de tecnologias em saúde. As notificações das não conformidades relacionadas à vigilância destas tecnologias aconteciam de forma descentralizada e, em grande parte, através de formulários impressos, dificultando a consolidação, análise, tratamento e a disseminação de informações. Além disso, a prática de notificar entre os colaboradores era pequena em relação à atividade assistencial existente. Objetivando a melhoria do processo de acompanhamento do comportamento das tecnologias em saúde foi desenvolvido na própria organização um sistema de notificação eletrônica, com a finalidade de ampliar o acesso dos colaboradores a esta atividade, estimular o crescimento do capital intelectual na prática de vigilância sanitária hospitalar, padronizar conceitos e consolidar uma visão sistêmica do tratamento das não conformidades, buscando a melhoria da qualidade do registro e quantidade das notificações realizadas.

PALAVRAS CHAVE: Vigilância Sanitária, Notificação, Tecnologia da Informação, Tecnologias Biomédicas.

\section{ABSTRACT}

The HCFMUSP Health System, as a part of the ANVISA Sentinel Network since 2001, undertook the commitment in post-marketing surveillance of health technologies. Notifications of noncompliance related to these technologies took place in a decentralized and largely through printed forms, hindering the consolidation, analysis, processing and dissemination of information. Furthermore, the practice of notifying among employees was small compared to care activities involvement. Aiming improvement of the process of monitoring the health technology utilization outcomes was developed in the organization a system of electronic notification, in order to increase the access of employees to this activity, stimulate the growth of intellectual capital in sanitary surveillance hospital standardized concepts and consolidate a systemic treatment of non-conformities, seeking to improve the quality and quantity of the record of the notifications made .

KEYWORDS: Surveillance, Notification, Information Technology, Biomedical

\footnotetext{
${ }^{1}$ Submetido em 30 de Outubro 2013. Aceito em 02 de abril de 2014. O artigo foi avaliado segundo o processo de duplo anonimato e avaliado pelo editor. Editores responsáveis: Márcio Augusto Gonçalves e Lucas Maia dos Santos. Reprodução parcial ou total e trabalhos derivativos permitidos com a citação apropriada da fonte.
} 


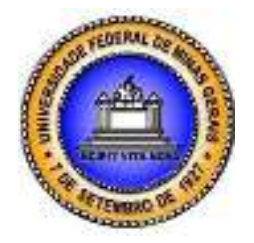

Technologies

\section{RESUMEN}

El Complejo HCFMUSP, por ser parte de la Red Sentinel de la ANVISA desde 2001, assumió el compromiso de colaborar en la vigilancia pos comercialización de tecnologías en salud. Las notificaciones de disconformidad de estas tecnologias solian hacerse de manera descentrizada y gran parte atraves de formularios impresos, lo que dificultaba la consolidación, análisis, tratamiento y diseminacion de informaciones. Ademas de eso, entre los colaboradores, la practica de notificar era pequeña en comparación con la actividad de asistencia. Con el objetivo de mejorar el proceso de acompañamento del comportamiento de las tecnologias fue desarrollado en la propria organizacion um sistema de notificacion electronica ,que tiene por finalidad ampliar el acceso de los colaboradores en esta actividad, estimulando el crescimiento del capital intelectual en la pratica de vigilancia sanitaria hospitalar.

PALABRAS CLAVE: Vigilancia Sanitaria, Notificación, Tecnología de la Información, Tecnología Biomédica

\section{INTRODUÇÃO}

A rapidez na incorporação de novas tecnologias, em todas as áreas de atuação da saúde, tem demonstrado a necessidade de fortalecer a vigilância pós-uso/ pós-comercialização destas tecnologias em saúde (ANVISA, s.d.).

Toda tecnologia em saúde só pode ser comercializada após receber autorização através de um número de registro emitido pela Agência Nacional de Vigilância Sanitária / Ministério da Saúde (Anvisa/MS), porém, se faz necessário um monitoramento da qualidade destas tecnologias liberadas para o comércio com fins diagnósticos e terapêuticos.

Entre as ações desenvolvidas pela Anvisa destaca-se o fortalecimento da vigilância pós-uso/ pós-comercialização das tecnologias em saúde, conhecida como VIGIPÓS, por meio da vigilância de eventos adversos (EA) e de queixas técnicas (QT), uma vez que estas ocorrências só serão conhecidas quando estes produtos forem utilizados em escala comercial.

A vigilância de EA e QT dentro de um estabelecimento de assistência a saúde se consolida através das notificações realizadas pelos profissionais que têm contato direto com os pacientes e/ou com tais tecnologias. Notificar EA e QT associados às tecnologias em saúde significa comunicar potenciais agravos à saúde dos pacientes ou usuários, efeitos inesperados ou indesejáveis, desvio da qualidade ou falha, entre outros fatos que comprometem a segurança sanitária do produto, ou seja, deve haver alguma suspeita da relação de causa e efeito entre a tecnologia utilizada e o incidente ocorrido.

Em cada unidade do Complexo HCFUMUSP existe um Gerente de Risco (GR) designado pela Diretoria Executiva (DIREX) que tem como principais atribuições assessorar a DIREX na elaboração e implantação de ações em Vigilância Sanitária, por meio do gerenciamento de informações em saúde e das notificações de não conformidades das tecnologias em saúde, e desenvolver atividades de prevenção, detecção, diminuição e eliminação de riscos, buscando oportunidades de melhoria nos processos assistenciais e administrativos, visando à segurança e proteção do paciente, do colaborador e da Instituição. Além disso, é ele quem atua como elo entre o HCFMUSP e a ANVISA. 

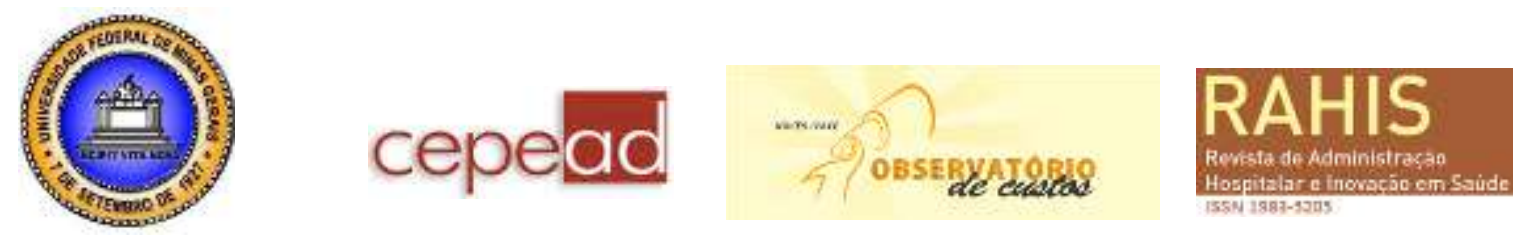

A Vigilância Sanitária Hospitalar do Núcleo de Informações em Saúde do Hospital das Clínicas da Faculdade de Medicina da Universidade de São Paulo - VISA-NIS/ HCFMUSP tem como objetivo a coordenação das ações de vigilância sanitária hospitalar, contribuindo para a redução de eventos que impactem nos objetivos estratégicos da organização, na qualidade e segurança da assistência de saúde prestada aos seus clientes e nos processos relacionados aos colaboradores, aos fornecedores e à sociedade, por meio de um enfoque estruturado e disseminado, alinhado à missão, visão e valores institucionais, com a finalidade de preservar, criar valor e conhecimento.

Uma das principais atribuições do VISA-NIS é estabelecer fluxos e processos para facilitar a identificação, a avaliação e o gerenciamento de informações relacionadas a não conformidades relacionadas às tecnologias em saúde.

Para fins de monitoramento das ocorrências, as tecnologias em saúde são agrupadas nas seguintes áreas: Tecnovigilância (equipamentos, materiais médico-hospitalares e kits diagnósticos), Farmacovigilância (medicamentos, vacinas, imunoglobulinas), Hemovigilância (sangue e seus componentes), Vigilância de Saneantes (produtos de limpeza, desinfecção e esterilização) e outros (ANVISA, 2002).

No HCFMUSP as notificações das não conformidades das tecnologias em saúde aconteciam de forma descentralizada e, em grande parte, através de formulários impressos, dificultando a consolidação, a análise, o tratamento e a disseminação das informações. Além disso, a prática de notificar entre os colaboradores ainda é pequena em relação à atividade assistencial prestada.

Objetivando a melhoria do processo de acompanhamento do comportamento das tecnologias em saúde foi desenvolvido na própria organização um sistema de notificação eletrônica. Sua finalidade foi ampliar o acesso dos colaboradores a essa atividade, disseminar o conhecimento teórico e prático da vigilância sanitária hospitalar, padronizar conceitos e consolidar uma visão sistêmica do tratamento das não conformidades, buscando a melhoria da qualidade do registro e quantidade das notificações realizadas.

Segundo Morais, Friedrich, Melchior et al, um benefício de destaque de um sistema de notificação eletrônica é que a rapidez e a precisão da notificação auxiliaria a tomada de medidas oportunas para impedir ou minimizar a ocorrência de agravos à saúde das populações expostas (MORAIS, FRIEDRICH, MELCHIOR et al, 2013).

\section{REFERENCIAL TEÓRICO}

Os benefícios das novas tecnologias em saúde são cada vez maiores e melhores, porém o seu desenvolvimento pode também implicar no aumento dos riscos que afetam a segurança, a saúde humana, a integridade profissional, o meio ambiente e a imagem institucional (ANVISA, 2006).

A Resolução da Diretoria Colegiada (RDC) no 02/2010 da ANVISA define tecnologias em saúde como sendo o conjunto de produtos para a saúde (equipamentos médicosodontológicos, medicamentos e insumos médico-hospitalares) e de procedimentos utilizados na prestação de serviços de saúde, bem como das técnicas de infraestrutura desses serviços e de sua organização (BRASIL, 2010).

Segundo a $\mathrm{RDC} \mathrm{n}^{\circ} 7$ a definição de risco é a probabilidade de ocorrência de um dano associado a sua gravidade (BRASIL, 2010).

De acordo com a Organização Mundial da Saúde (OMS, 2003):

Nenhum rigor no processo de revisão de pré-comercialização pode prever todas as 

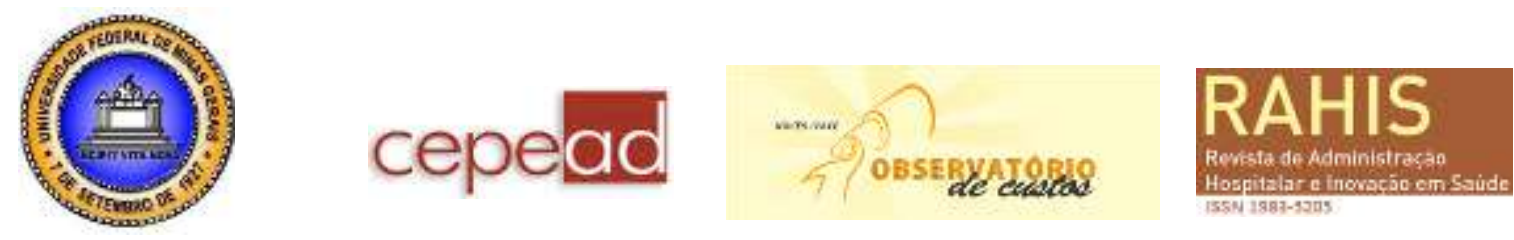

falhas ou incidentes em produtos médicos decorrentes do mau uso. É por meio do uso real que os problemas não previstos relacionados à segurança e ao desempenho podem ocorrer.

Com o intuito de acompanhar o comportamento das tecnologias em saúde, na fase póscomercialização, a Anvisa criou a Rede Sentinela, inicialmente na forma de projeto e hoje já firmada como rede, que representou um importante avanço na segurança dentro do ambiente hospitalar (ANVISA, s.d.).

A Rede Sentinela é composta por serviços de saúde que tem como objetivo a ampliação e a sistematização da vigilância de tecnologias em saúde utilizadas na atenção à saúde, visando garantir aos pacientes e profissionais o uso de produtos com qualidade (ANVISA, 2001).

O HCFMUSP faz parte da Rede Sentinela desde 2001, assumindo o compromisso de colaborar com a vigilância destas tecnologias. Em 2011, esta parceria foi reafirmada e todas suas unidades hospitalares, como também as unidades parceiras, foram incluídas na rede (BRASIL, 2012).

Para coordenar as atividades relacionadas à vigilância das tecnologias dentro dos estabelecimentos de saúde, cada unidade do Complexo HCFUMUSP nomeou um Gerente de Risco (GR) através da Diretoria Executiva (DIREX). Conforme a RDC no 02/2010, Gerenciamento de Riscos é a aplicação sistemática de políticas de gestão, procedimentos e práticas na análise, avaliação, controle e monitoramento de risco (BRASIL, 2010).

O monitoramento dos EA e das QT relacionadas às tecnologias em saúde se dá através das notificações realizadas pelos profissionais que têm contato direto com os pacientes e/ou com tais tecnologias. Entende-se por EA as ocorrências com tecnologias em saúde que causaram danos a saúde de pacientes ou de funcionários, e por QT, as ocorrências verificadas com as tecnologias em saúde antes de serem utilizadas na assistência (BRASIL, 2006).

Partindo-se do princípio que os pacientes não devam sofrer danos, a questão da segurança tem assumido destaque na qualidade da assistência em vários países. Pesquisas mostram que os EAs afetam em média $10 \%$ das admissões hospitalares e representam um dos maiores desafios para a qualidade na área da saúde (GALLOTTI, 2003). A ausência de segurança durante a assistência a saúde pode acarretar, no prolongamento da internação, aumento nos custos da hospitalização, necessidade de tratamentos adicionais, exames e procedimentos extra, podendo até levá-los a morte (SANTOS, 2007).

Como base juridica e funcional para as atividades desenvolvidas pelo GR, a Portaria Interministerial $n^{\circ} 2.400$ de 2007, que estabelece os requisitos para certificação de unidades hospitalares como Hospitais de Ensino, instituiu que fossem constituídas e mantidas em permanente funcionamento, as atividades de Hemovigilância, Farmacovigilancia e Tecnovigilância (BRASIL, 2007). Além disso, visando promover melhorias relativas à segurança do paciente de forma a prevenir e reduzir a incidência de eventos adversos no atendimento e internação, o MS lançou o Programa Nacional de Segurança do Paciente (BRASIL, 2013).

Sabe-se que atualmente os hospitais, para melhorar a eficiência do monitoramento da qualidade, têm implementado sistemas de vigilância eletrônica, aonde através da automação os serviços de saúde vêm substituindo o controle passivo pela vigilância ativa, que permite intervenções simultâneas e melhoria da qualidade e segurança (TINOCO, EVANS, STAES et al, 2011).

\section{ASPECTOS METODOLÓGICOS}



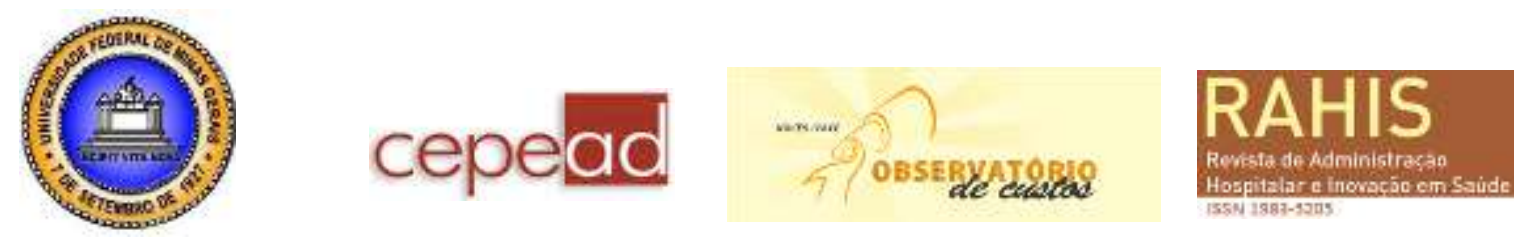

O estudo em questão utilizou como técnica metodológica a observação participante, através da estratégia entre pesquisa ação e pesquisa documental.

O trabalho foi realizado no Hospital das Clínicas da Faculdade de Medicina da Universidade de São Paulo - HCFMUSP, localizado na zona Oeste do município de São Paulo, que é caracterizado como hospital de ensino universitário, autarquia estadual, nível terciário de atenção à saúde, composto de sete institutos e dois hospitais auxiliares, 63 salas cirúrgicas, 2.074 leitos instalados, 67.039 entradas hospitalares, 32.848 cirurgias realizadas, 1.493.391 consultas ambulatoriais, 323.534 atendimentos de emergência, 12.084.395 exames diagnósticos, 66.418 saídas hospitalares ${ }^{1}$, além de ser referência para a realização de procedimentos de alta complexidade como: transplantes de fígado, rins, pulmão, coração, medula óssea, etc.

A construção da ferramenta de notificação eletrônica de não conformidade de tecnologias em saúde pós-comercialização, nomeada NOTIFICA HC, foi desenvolvida integralmente por núcleos próprios de gestão da informação do HCFMUSP. Neste aspecto, foram envolvidos no desenvolvimento da ferramenta de notificação o VISA-NIS que se responsabilizou pela pesquisa e estabelecimento de campos mínimos necessários de forma a obter aderência do colaborador, pela implantação e treinamento dos usuários do sistema e o Núcleo Especializado em Tecnologia da Informação (NETi) que se incumbiu do desenvolvimento de ferramentas sistêmicas de acesso, e criação de um banco de dados relacional que permitisse a integração da funcionalidade aos demais sistemas internos da Organização. O conteúdo foi baseado nos formulários (impressos) então existentes na instituição e atualizado com base nos critérios e formulários da Anvisa (sistema NOTIVISA).

Dentre as principais vantagens do NOTIFICA HC pode se citar a utilização do meio eletrônico, de forma a garantir a acessibilidade uma vez que estará disponibilizada na Intranet institucional. A facilidade de preenchimento, a racionalização das informações através de campos mínimos obrigatórios, o monitoramento imediato das informações, a padronização de conceitos, a troca de informações entre os institutos e hospitais do Complexo HCFMUSP e a disseminação da informação, também compõem o rol de vantagens da ferramenta.

A ferramenta está sendo implantada progressivamente nas unidades do HCFMUSP. Sua implantação começou com o projeto piloto no Instituto de Radiologia - InRad em abril de 2012. Atualmente já está em uso no InRad, no Hospital Auxiliar do Cotoxó - HAC, no Instituto de Psiquiatria - IPq e no Instituto de Ortopedia - IOT. O prazo para o término da implantação em todos os Institutos e Hospitais do Complexo é julho de 2013.

\section{RESULTADOS OBTIDOS}

A ferramenta disponível na intranet, através da logomarca (Figura 1) criada pelo VISA-NIS e formatada em parceria com o Núcleo de Comunicação Institucional (NCI), é constituída de campos com listbox e campos abertos e a média de tempo para preenchimento verificado durante os treinamentos foi de 10 minutos (Figura 2).

A Tabela 1 apresenta a quantidade de notificações e sua distribuição por tipo (EA e QT) para as quatro unidades do Complexo HCFMUSP que utilizam o sistema NOTIFICA $\mathrm{HC}$, enquanto as demais unidades estão em fase de implantação.

A partir do sistema NOTIFICA HC será possível obter um banco de dados institucional para a busca de oportunidades de melhorias nos processos que tangenciam a segurança do paciente e do colaborador. Entretanto, toda e qualquer notificação de não conformidade confirmada pelo GR é registrada no sistema NOTIVISA da ANVISA. A 
aceitação, nestas quatro unidades, foi considerada significativa, pois desde o início já foram 1.303 notificações feitas através do sistema eletrônico.

Figura 1 - Logomarca do NOTIFICA HC na intranet - acesso à ferramenta de notificação eletrônica

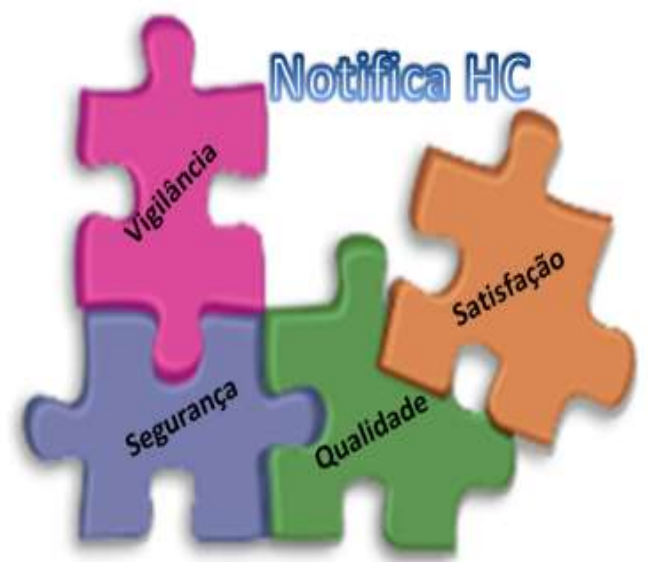

1. Dados Monitoramento de Indicadores do NIS HCFMUSP, 2012

Figura 2 - NOTIFICA HC - Ficha para Notificação de Não Conformidade disponibilizada na intranet institucional 


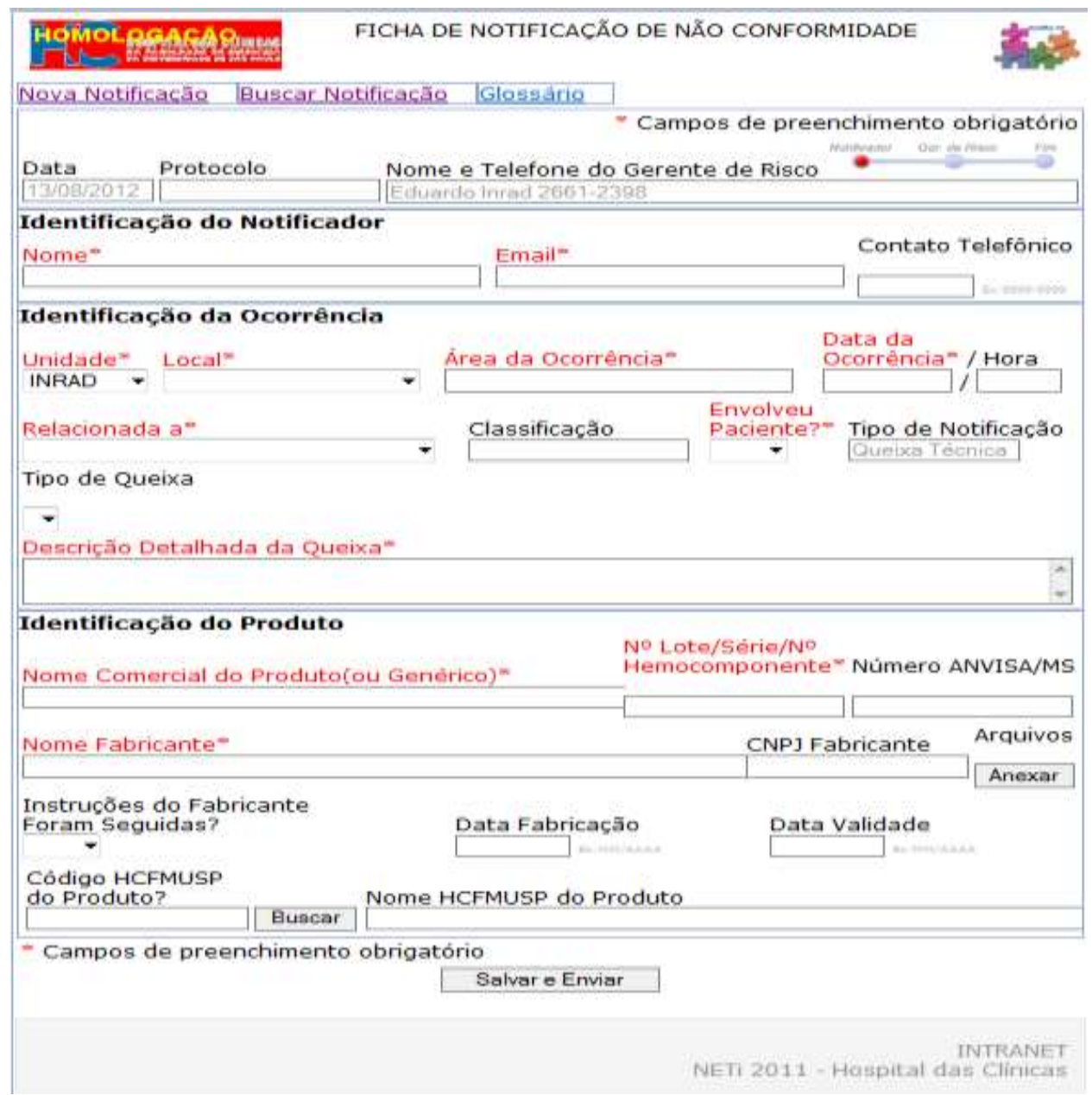

Tabela 1 - Notificações de não conformidades do NOTIFICA HC por Instituto, por Área de Vigilância e por Tipo (Evento Adverso e Queixa Técnica), de abril de 2012 a abril de 2013

\begin{tabular}{|l|l|l|l|l|l|}
\hline \multicolumn{1}{|c|}{ Notificações } & \multicolumn{1}{c|}{ InRad } & \multicolumn{1}{c|}{ IPq } & \multicolumn{1}{c|}{ IOT } & \multicolumn{1}{c|}{ HAC } & \multicolumn{1}{c|}{ Total } \\
\hline Farmacovigilância & 250 & 71 & 85 & 252 & 658 \\
\hline Tecnovigilância & 345 & 29 & 226 & 26 & 626 \\
\hline Hemovigilância & 0 & 0 & 0 & 0 & 0 \\
\hline Vigilância de Saneantes & 2 & 1 & 1 & 4 & 8 \\
\hline Cosméticos e Higiene & 2 & 2 & 7 & 0 & 11 \\
\hline Evento Adverso (EA) & 266 & 21 & 43 & \multicolumn{1}{|c|}{1} & 331 \\
\hline Queixa Técnica (QT) & 333 & 82 & 276 & 281 & 972 \\
\hline Total & $\mathbf{5 9 9}$ & $\mathbf{1 0 3}$ & $\mathbf{3 0 3}$ & $\mathbf{2 7 3}$ & $\mathbf{1 . 3 0 3}$ \\
\hline
\end{tabular}




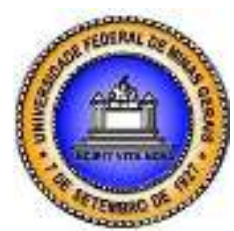

Os Gráficos 1 e 2 , a seguir, apresentam a quantidade de notificações e sua distribuição por tipo (EA e QT) de todo o Complexo HCFMUSP. Todos os Institutos e Hospitais do Complexo HCFMUSP notificam as não conformidades de tecnologias em saúde póscomercializadas no sistema NOTIVISA da ANVISA.

Gráfico 1 - Total de Notificações realizadas no HCFMUSP (sistema eletrônico e impresso) de janeiro de 2011 a fevereiro de 2013 e notificadas no NOTIVISA

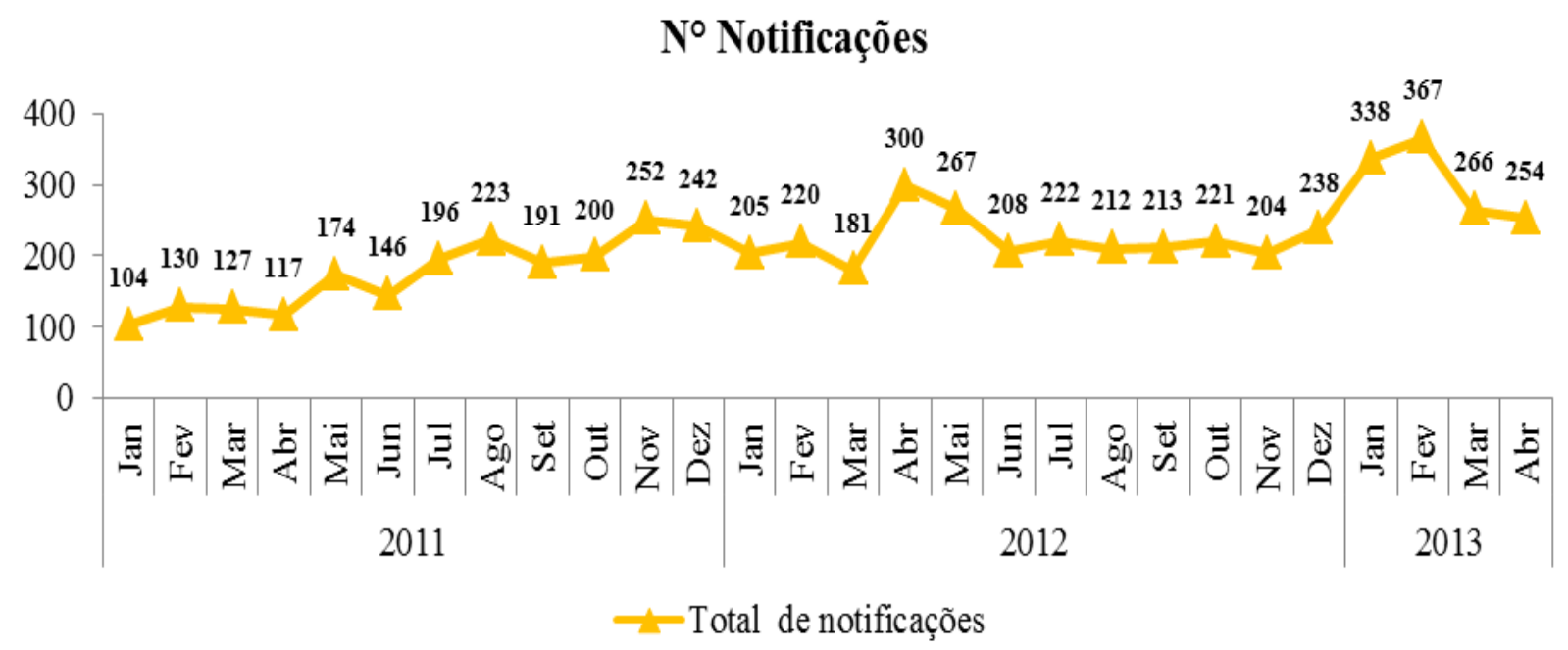

Gráfico 2 - Total de Notificações realizadas no HCFMUSP (sistema eletrônico e impresso) por tipo (Evento Adverso e Queixa Técnica), de janeiro de 2011 a fevereiro de 2013, notificadas no NOTIVISA

Tipo Notificação - HCFMUSP

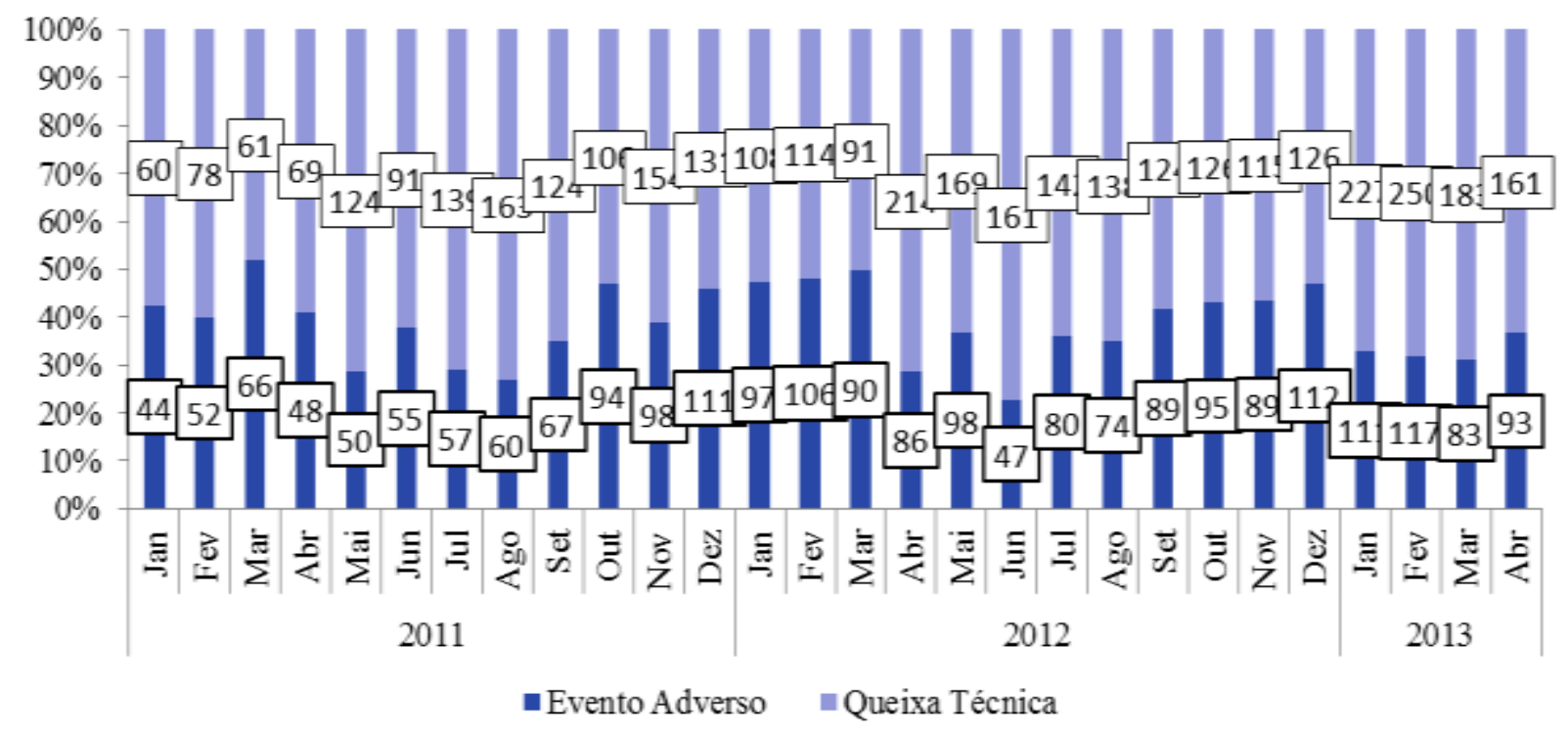




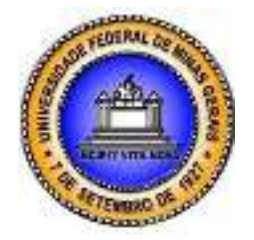

\section{CONCLUSÃO}

Apesar da ferramenta ainda não estar disponibilizada em todo o Complexo HCFMUSP, foi possível notar que houve maior adesão dos colaboradores e com isto aumento do número das notificações nos institutos implantados, totalizando 1.303 notificações realizadas através do sistema eletrônico, no período de abril/2012 a abril/2013. Além disso, devido à uniformidade de preenchimento foi possível criar um banco de dados institucional que permitirá estabelecer protocolos de tratamento das não conformidades levantadas. A disponibilização da ferramenta na Intranet institucional possibilitou maior acessibilidade, facilidade de preenchimento, racionalização das informações através de campos mínimos obrigatórios, monitoramento imediato das informações, padronização de conceitos, troca e disseminação de informações entre os institutos e hospitais do Complexo.

Alguns aspectos limitantes encontrados foram barreiras enfrentadas para realização de treinamento de colaboradores dos institutos que implantaram a ferramenta NOTIFICA HC devido à dificuldade de agendamento de espaço físico para treinamento de multiplicadores e disponibilidade das equipes, que necessitariam ficar afastadas do setor de trabalho durante o treinamento. Além disso, como em toda implantação de algo novo, há sempre o enfrentamento de resistência pela substituição de rotinas já estabelecidas.

O presente relato limitou-se a descrever a implantação de um sistema eletrônico de notificação de não conformidades relacionadas às tecnologias em saúde, mostrando que este tipo ação corrobora com a vigilância pós-comercialização e desta forma com a segurança do paciente, do profissional da saúde e da instituição.

Identificou-se a necessidade de desenvolvimento de novas pesquisas com o objetivo de avaliar o impacto das notificações de não conformidades para a instituição e o perfil epidemiológico dos eventos adversos causados por estas não conformidades.

\section{REFERÊNCIA BIBLIOGRÁFICA}

ANVISA (Brasil). Pós-Comercialização Pós-Uso, Brasília, [s.d.]. Disponível em: <http://portal.anvisa.gov.br/wps/content/Anvisa+Portal/Anvisa/Pos+-+Comercializacao++Pos+-+Uso/Vigipos> . Acesso em: 01 jun. 2013.

ANVISA (Brasil). Boletim Informativo ANVISA nº11, Brasília, 2001. Disponível em: <http://www.anvisa.gov.br/divulga/public/boletim/11_01.pdf>. Acesso em: 01 jun. 2013.

ANVISA (Brasil). Boletim Informativo ANVISA n²4, Brasília, 2002. Disponível em: <http://www.anvisa.gov.br/divulga/public/boletim/24_02.pdf>. Acesso em: 01 jun. 2013.

ANVISA (Brasil). Diretrizes Nacionais de Vigipós, Brasília, 2006. Disponível em: <http://portal.anvisa.gov.br/wps/content/Anvisa+Portal/Anvisa/Pos+-+Comercializacao++ Pos+-+Uso/Vigipos/Assunto+de+Interesse/Diretrizes+Nacionais+de+VIGIPOS >. Acesso em: 01 jun. 2013. 
ANVISA (Brasil). Histórico, Brasília, [s.d.]. Disponível em: $<$ http://portal.anvisa.gov.br/wps/content/Anvisa+Portal/Anvisa/Pos+-+Comercializacao++Pos+-+Uso/Rede+Sentinela/Assunto+de+Interesse/Historico>. Acesso em: 01 jun. 2013.

BRASIL. Agência Nacional de Vigilância Sanitária. Anexo I da Portaria $n^{\circ}$ 354. Brasília, DF, Brasil, 11 ago. 2006.

BRASIL. Ministério da Saúde/Ministério da Educação. Portaria Interministerial $\mathrm{n}^{\circ} 2.400$. Brasília, DF, Brasil, 02 out. 2007.

BRASIL. Agência Nacional de Vigilância Sanitária. Resolução - RDC $n^{o}$ 2. Brasília, DF, Brasil, 25 jan. 2010.

BRASIL. Agência Nacional de Vigilância Sanitária. Resolução - RDC $n^{o} 7$. Brasília, DF, Brasil, 24 fev. 2010.

BRASIL. Portaria n ${ }^{o}$ 523. Ministério da Saúde. Brasília, DF, Brasil, 04 abr. 2012.

BRASIL. Portaria n ${ }^{o}$ 529. Ministério da Saúde. Brasília, DF, Brasil, 01 abr. 2013.

GALLOTTI, R.M. Eventos adversos e óbitos hospitalares em serviço de emergências clínicas de um hospital universitário terciário: um olhar para a qualidade da atenção. Tese (Doutorado) - Faculdade de Medicina da Universidade de São Paulo, São Paulo, 2003.

MORAIS, L.D.O., FRIEDRICH, K., MELCHIOR, S.C., et al. Eventos adversos e queixas técnicas relacionados ao fio para sutura cirúrgica comercializado no Brasil. Vigilância Sanitária em Debate 1, Rio de Janeiro, 2013.

WORLD HEALTH ORGANIZATION. Medical device regulations : global overview and guiding $\quad 2003 . \quad$ Drinciples, 2 Disponível http://www.who.int/medical_devices/publications/en/MD_Regulations.pdf. Acesso em: 18 mai. 2013.

SANTOS, J.O. Sentimento de profissionais após ocorrência de erros de medicação. Acta Paulista, 483-8, São Paulo, 2007.

TINOCO, A., EVANS, R.S., STAES, C.J., LOYD, J.F., ROTHSCHILD, J.M., et al. Comparison of computerized surveillance and manual chart review for adverse events. Journal of the American Medical Informatics Association, 18(4), 491-497, 2011. 